\title{
Very Strong TeV Emission as Gamma-Ray Burst Afterglows
}

\author{
Tomonori Totani \\ Department of Physics, School of Science, The University of Tokyo, Tokyo 113-0033, Japan \\ E-mail: totani@utaphp2.phys.s.u-tokyo.ac.jp
}

\begin{abstract}
Gamma-ray bursts (GRBs) and following afterglows are considered to be produced by dissipation of kinetic energy of a relativistic fireball and radiation process is widely believed as synchrotron radiation or inverse Compton scattering of electrons. We argue that the transfer of kinetic energy of ejecta into electrons may be inefficient process and hence the total energy released by a GRB event is much larger than that emitted in soft gamma-rays, by a factor of $\sim\left(m_{p} / m_{e}\right)$. We show that, in this case, very strong emission of $\mathrm{TeV}$ gamma-rays is possible due to synchrotron radiation of protons accelerated up to $\sim 10^{21} \mathrm{eV}$, which are trapped in the magnetic field of afterglow shock and radiate their energy on an observational time scale of $\sim$ day. This suggests a possibility that GRBs are most energetic in $\mathrm{TeV}$ range and such $\mathrm{TeV}$ gamma-rays may be detectable from GRBs even at cosmological distances, i.e., $z \sim 1$, by currently working ground-based telescopes. Furthermore, this model gives a quantitative explanation for the famous long-duration $\mathrm{GeV}$ photons detected from GRB940217. If $\mathrm{TeV}$ gamma-ray emission which is much more energetic than GRB photons is detected, it provides a strong evidence for acceleration of protons up to $\sim 10^{21} \mathrm{eV}$.
\end{abstract}

Subject headings: acceleration of particles - gamma rays: bursts - gamma rays: theory

\section{Introduction}

Gamma-ray bursts (GRBs) are widely believed as dissipation of kinetic energy of relativistic motion produced by an expanding fireball with a Lorentz factor of $\sim 10^{2}-10^{3}$ (see e.g., Piran 1994 for a review). The recently discovered afterglows following GRBs are also considered as similar phenomena, which are dissipation in the external shock generated by the collision with interstellar matter (Paczyński \& Rhoads 1993; Katz 1994; Mészáros 
\& Rees 1997; Vietri 1997a). The cosmological origin of GRBs is now almost confirmed by the discovery of metal absorption lines at $z=0.835$ for the optical afterglow of GRB970508 (Metzger et al. 1997), and some of observations for X-ray, optical, and radio afterglows are in rough agreement with the prediction of the cosmological fireball model (Wijers, Rees, Mészáros 1997; Waxman 1997a, b; Vietri 1997b). However, there is large variation in the afterglow response of GRBs (e.g., Groot et al. 1998), and it is not yet clear whether the simple afterglow model is applicable for all of GRBs.

There are two important, but highly uncertain parameters in such theoretical models of GRBs and afterglows: the degree of equipartition between the internal energy of shock heated matter and magnetic fields $\left(\xi_{B}\right)$ and between protons and electrons $\left(\xi_{e}\right)$. In most of publications which calculated model predictions of GRBs or afterglows, these two parameters are assumed to be of order unity, and the radiation process is considered as electron synchrotron (or inverse Compton scattering). In this case the efficiency of energy release in GRBs or afterglows compared to the total energy of a GRB event $(E)$ is of order unity. However, currently there is no clear evidence for efficient energy transfer into electrons and magnetic fileds, although some of observational data are consistent with $\xi_{e} \sim 1$ (Waxman 1997a). If the energy transfer from protons into electrons is inefficient, energy stored in electrons is only a fraction of $\xi_{e} \sim\left(m_{e} / m_{p}\right)$ of the total fireball energy and hence about 2000 times larger energy must be released as kinetic energy of relativistic ejecta than the observed energy emitted as GRB photons. The GRB photon energy is $\sim 10^{51} \mathrm{erg}$ if the radiation is isotropic and the redshift of most distant GRBs, $z_{\max } \sim 1$. Then the total energy $E$ may be uncomfortably large because most of GRB models are based on gravitational collapses of massive stars in which available energy is $\sim 3 \times 10^{53}$ erg and most of this energy will be lost as neutrinos. However, the theoretical estimate of merger rate of binary neutron stars is about $10^{2-3}$ times higher than the observed GRB rate (Lipunov et al. 1995; Totani 1997,1998), which suggests that GRB is strongly beamed if GRBs are associated to merger of binary neutron stars (Blinnikov et al. 1984). If GRBs are actually beamed with such a strong beaming factor, the above constraint of energy budget becomes much weaker. Much more energetic models of GRBs have also been proposed such as the microquasar model, in which total energy of $\sim 10^{54}$ erg can be supplied to a fireball (Paczyński 1998).

In this letter we argue that time scale of energy transfer into electrons by the Coulomb interactions is much larger than the expansion time of external shock, while magnetic field may achieve the equipartition with protons in the shock heated matter. We then show that, as a consequence of this scenario, a very strong $\mathrm{TeV}$ emission is expected during a few days after GRBs by synchrotron radiation of $10^{20} \mathrm{eV}$ protons and it may be detectable by current ground-based telescopes even from a GRB at cosmological distances, in spite of 
significant attenuation due to $e^{ \pm}$creation with intergalactic infrared photons. Synchrotron emission of protons of $\sim 10^{20} \mathrm{eV}$ from GRBs was first considered by Vietri (1997c), and Böttcher \& Dermer (1998) extended the analysis to emission from afterglows. Both papers concluded that TeV gamma-rays are detectable only for nearby GRBs $(z \lesssim 0.1)$, assuming that total fireball energy is of the same order with that of GRB photons. The natural units with $c=\hbar=1$ is used in this letter.

\section{Efficiency of Energy Transfer into Electrons and Magnetic Fileds}

The evolution of external shock is described by $b E=16 \pi n m_{p} r^{3} \gamma^{2} / 17$, where $E$ is the total energy released in an opening angle of $\Delta \Omega, b(=4 \pi / \Delta \Omega)$ a beaming factor, and $\gamma$ the Lorentz factor of the shock heated matter (Blandford \& McKee 1976). The location of the shock, $r$, is measured in the laboratory frame and $n$ is the (unshocked) interstellar matter density. Initially the kinetic energy stored in electrons is only a fraction of $\left(m_{e} / m_{p}\right)$ of the total energy, and much greater energy of protons must be efficiently transferred into electrons by some interactions in the shock heated matter in order to achieve energy equipartition between electrons and protons. However, relative importance of the Coulomb interaction becomes smaller with increasing energy of particles, and the particle energy in relativistic shocks is much greater than that in non-relativistic shocks such as supernova remnants. The time scale of energy transfer in relativistic plasma is difficult to estimate accurately, but a rough estimate is given by $\tau_{e p} \sim\left(n^{\prime} \sigma_{t}\right)^{-1}$, where $n^{\prime}=4 \gamma n$ is the proton number density of the shocked matter measured in the shocked-shell frame and $\sigma_{t}=4 \pi L_{e}\left(e^{2} / m_{e} \gamma\right)^{2}$ is the transport cross section for electron-proton collisions. The Coulomb logarithm is given by $L_{e}=\ln \left(a m_{e} \gamma\right)$, where $a=\left(m_{e} \gamma / 4 \pi n^{\prime} e^{2}\right)^{1 / 2}$ is the Debye length (e.g., Lifshitz \& Pitaevskii 1981). This time scale should be compared to the expansion time measured in the shell frame, $r / \gamma$, and we find $\tau_{e p} /(r / \gamma)=1.1 \times 10^{4} b^{-1 / 3} E_{51}^{-1 / 3} n_{1}^{-2 / 3} \gamma^{8 / 3}$, where $E=10^{51} E_{51}$ erg and $n=n_{1} \mathrm{~cm}^{-3}$. Hence energy transfer through the Coulomb interaction is likely to be inefficient.

On the other hand, magnetic field in the shocked matter may be in equipartition with the random motion energy of protons which is directly converted from the kinetic energy of a fireball. Recall that, in the well-known equation of magnetohydrodynamics, the time evolution of magnetic field, $\partial \mathbf{B} / \partial t$, is governed by the diffusion term and the source term, $\operatorname{rot}[\mathbf{u} \times \mathbf{B}]$, where $\mathbf{u}$ is the velocity field of fluid. In afterglow shocks there will be turbulent motion of $\mathbf{u}^{2} \sim 1$ and a coherent length scale will be smaller than the shell thickness of the shocked matter measured in the shell frame, $\sim r / \gamma$. This suggests that $|\operatorname{rot}[\mathbf{u} \times \mathbf{B}]| \gtrsim(\gamma / r) B$ and hence the growth time scale of magnetic field may be $\lesssim r / \gamma$. 
Since the expansion time is also $\sim r / \gamma$ in the shell frame, it is possible that equipartition between magnetic field and protons is achieved while electrons carry much smaller energy. Although the above argument is quite rough and some unknown processes in relativistic matter may allow electrons to be in equipartition with protons, it seems rather reasonable to consider the case of $\xi_{B} \sim 1$ and $\xi_{e} \sim\left(m_{e} / m_{p}\right) \ll 1$. In order to investigate such an energetic model of GRBs, we use $E=10^{52} E_{52}$ erg and $b=200 b_{200}$ as typical values, with which $E$ is about 2000 times larger than the energy emitted in GRB photons, i.e., $\sim 10^{51} b^{-1}$ erg when $z_{\max } \sim 1$.

\section{Proton Synchrotron in Afterglow Shock}

If the energy transfer from protons into electrons is inefficient but magnetic field is nearly in equipartition, the synchrotron radiation of protons becomes relatively important. The energy density of shocked matter is given by $4 \gamma^{2} n m_{p}$ in the shell frame and magnetic field can be written as $B=\left(32 \pi \xi_{B} \gamma^{2} n m_{p}\right)^{1 / 2}$. It has been considered that protons may be accelerated up to $\sim 10^{20} \mathrm{eV}$ in GRBs because the physical quantities of GRBs allow acceleration of protons to such high energies and observed flux of highest energy cosmic rays is consistent with the GRB occurrence rate provided that such protons carry roughly the same amount of energy with GRBs (Waxman 1995; Vietri 1995). We assume that the shock acceleration time is given by $\eta r_{L}$, where $r_{L}=m_{p} \gamma_{p} /(e B)$ is the Larmor radius, $\gamma_{p}$ is the proton Lorentz factor in the shell frame, and $\eta$ is a parameter of order unity. The maximum energy obtained in the external shock is given by the equation $\eta r_{L}=r / \gamma$, and we find $4.21 \times 10^{21} \eta^{-1} \xi_{B}^{1 / 2} n_{1}^{1 / 6} \gamma_{100}^{1 / 3} b_{200}^{1 / 3} E_{52}^{1 / 3} \mathrm{eV}$ in the observer's frame, where $\gamma_{100}=\gamma_{0} / 100$ and $\gamma_{0}$ is the initial fireball Lorentz factor. On the other hand, the maximum energy is also constrained by synchrotron cooling. The cooling time at the shell frame is $t_{\mathrm{syn}}=3 m_{p}^{3} /\left(4 \sigma_{T} m_{e}^{2} U_{\mathrm{mag}} \gamma_{p}\right)$, where $U_{\mathrm{mag}}$ is the energy density of magnetic field. From the equation $\eta r_{L}=t_{\text {syn }}$ we then find the maximum energy in the observer's frame as $3.27 \times 10^{21} \eta^{-1 / 2} \xi_{B}^{-1 / 4} n_{1}^{-1 / 4} \gamma_{100}^{1 / 2} \mathrm{eV}$, which does not depend on the total fireball energy of GRBs. Therefore protons may by accelerated up to $10^{21-22} \mathrm{eV}$ for $\gamma_{0}=100-1000$, which is about one order of magnitude greater than the estimate obtained with $b E \sim 10^{51} \mathrm{erg}$ (Waxman 1995; Vietri 1995).

The protons accelerated up to $\sim 10^{21} \mathrm{eV}$ will radiate their energy by synchrotron radiation in magnetic fields of afterglow shock. The synchrotron photon energy in the observer's frame is given by $\varepsilon_{\gamma}=\gamma \gamma_{p}^{2} e B / m_{p}=2.8 \varepsilon_{p, 21}^{2} \xi_{B}^{1 / 2} n_{1}^{1 / 2} \mathrm{TeV}$, where $\varepsilon_{p, 21}=m_{p} \gamma \gamma_{p} /\left(10^{21} \mathrm{eV}\right)$ is proton energy in the observer's frame. Therefore synchrotron photon energy is related to proton energy independently of time and will extend to $\gtrsim$ 
TeV. Next let us check whether such protons can be confined in the afterglow shock. The ratio of Larmor radius of protons to the restframe shell thickness $r / \gamma$ is found as $r_{L} /(r / \gamma)=0.26 \xi_{B}^{-3 / 4} n_{1}^{-3 / 8} b_{200}^{-3 / 8} E_{52}^{-3 / 8} \varepsilon_{\gamma, \mathrm{TeV}}^{1 / 2} t_{\mathrm{day}}^{1 / 8}$, where $\varepsilon_{\gamma}=\varepsilon_{\gamma, \mathrm{TeV}} \mathrm{TeV}$ and the observation time is $t_{\text {day }}$ day $=r /\left(2 \gamma^{2}\right)$ or $\gamma=15 t_{\text {day }}^{-3 / 8} b_{200}^{1 / 8} E_{52}^{1 / 8} n_{1}^{-1 / 8}$. The transverse width of beamed shell is larger than the shell thickness unless the beaming factor is extremely large as $b>7.21 \times 10^{3} t_{\text {day }}^{-1} E_{52}^{1 / 3} n_{1}^{-1 / 3}$. Therefore the protons which correspond to synchrotron photons of $\lesssim \mathrm{TeV}$ can be trapped within the magnetic field of afterglow shock on a time scale of $\sim$ day. The cooling time observed on the Earth is related to the restframe cooling time as $t_{\text {syn,obs }}=t_{\text {syn }} /(2 \gamma)$, and we find $t_{\text {syn,obs }}=1.3 \xi_{B}^{-3 / 4} n_{1}^{-1 / 2} \varepsilon_{\gamma, \mathrm{TeV}}^{-1 / 2} t_{\text {day }}^{3 / 4} b_{200}^{-1 / 4} E_{52}^{-1 / 4}$ day. If the spectrum of accelerated protons is that of the standard shock acceleration theory, i.e., $d N_{p} / d \gamma_{p} \propto \gamma_{p}^{-\alpha}$ with $\alpha \sim 2$, luminosity of synchrotron radiation per decade of photon energy, $L\left(\varepsilon_{\gamma}\right) \equiv \varepsilon_{\gamma} d L / d \varepsilon_{\gamma}$, is proportional to $\varepsilon_{\gamma}^{\beta}$ with $\beta=(3-\alpha) / 2 \sim 0.5$. Hence the synchrotron emissivity becomes maximum at the cut-off energy and the above results suggest the following picture: protons accelerated to $\sim 10^{20-21} \mathrm{eV}$ will be trapped in afterglow shock and radiate most of their energy in the $\mathrm{TeV}$ range within a time scale of a few days. Since the total energy of protons could be comparable to the total kinetic energy of a fireball while electrons carry much smaller energy, the energy radiated by proton synchrotron around $\mathrm{TeV}$ range would be much larger than the energy released as GRB photons.

Now we proceed to estimate of the luminosity of proton synchrotron radiation. Suppose that accelerated protons in shocked matter have a power-law spectrum $(\alpha=2)$ in the range $\gamma_{0}^{2} \leq \tilde{\gamma}_{p} \leq \gamma_{u} \sim 10^{12}$ and total kinetic energy carried by them in the observer's frame is $\xi_{p} E$ :

$$
\frac{d N_{p}}{d \tilde{\gamma}_{p}}=\frac{\xi_{p} E}{m_{p} \ln \left(\gamma_{u} / \gamma_{0}^{2}\right)} \tilde{\gamma}_{p}^{-2},
$$

where $\tilde{\gamma}_{p}=\gamma \gamma_{p}$ is the proton Lorentz factor at the observer's frame and $\xi_{p} \sim 1$ if the accelerated protons are in equipartition. The observed luminosity is given by

$$
L\left(\varepsilon_{\gamma}\right) \equiv \varepsilon_{\gamma} \frac{d L}{d \varepsilon_{\gamma}}=2 \gamma^{2} \varepsilon_{\gamma} \frac{d \gamma_{p}}{d \varepsilon_{\gamma}} \frac{d N_{p}}{d \gamma_{p}} j_{\mathrm{syn}, \mathrm{p}},
$$

where $j_{\text {syn,p }}=4 \sigma_{T} m_{e}^{2} U_{\text {mag }} \gamma_{p}^{2} /\left(3 m_{p}^{2}\right)$ is the synchrotron energy loss rate of a proton in the shell frame. After some calculations we find

$$
L\left(\varepsilon_{\gamma}\right)=2.5 \times 10^{45} \xi_{p} \xi_{B}^{3 / 4} E_{52}^{5 / 4} b_{200}^{1 / 4} n_{1}^{1 / 2} t_{\text {day }}^{-3 / 4} \varepsilon_{\gamma, \mathrm{TeV}}^{1 / 2} \operatorname{erg~s}^{-1}
$$

where we have assumed $\left(\gamma_{0}, \gamma_{u}\right)=\left(10^{2}, 10^{12}\right)$. If we observe this emission from a distance of $d=3000 d_{3} \mathrm{Mpc}(z \sim 1)$, then the observed flux above $1 \mathrm{TeV}$ is $5.9 \times 10^{-10} \xi_{p} \xi_{B}^{3 / 4} E_{52}^{5 / 4} b_{200}^{5 / 4} n_{1}^{1 / 2} t_{\text {day }}^{-3 / 4} d_{3}^{-2}$ photons $\mathrm{cm}^{-2} \mathrm{sec}^{-1}$. This flux is further attenuated 
by the $e^{ \pm}$creation with intergalactic infrared photon field. The current estimate of the optical depth for this intergalactic absorption is still highly uncertain, but a typical value for TeV gamma-rays is $\tau \sim 10\left(e^{\tau}=2.2 \times 10^{4}\right)$ for $z \sim 1$ (Salamon \& Stecker 1998). The amount of infrared background is related to the amount of stars in the universe and this is uncertain by a factor of about 2. A factor of 2 reduction of the estimate of $\tau$ results in the attenuation of $e^{\tau} \sim 150$. Therefore the above flux would be attenuated by a factor of at least 100, and the attenuated flux is consistent with the upper limits set by the Whipple telescope (Connaughton et al. 1997) for some GRBs, which are about $10^{-10}-10^{-9} \mathrm{~cm}^{-2} \mathrm{sec}^{-1}$ depending on the source position in the field of view $\left(\sim 3^{\circ}\right)$. However, if a burst location is determined as well as some of recent GRBs for which afterglows are detected, and observation is made for a time scale of day, a flux of $\sim 10^{-12} \mathrm{~cm}^{-2} \mathrm{sec}^{-1}$ is detectable by currently working ground-based air Čerenkov telescopes (see, e.g., Kifune 1996 for a general review), and hence the $\mathrm{TeV}$ photons from GRBs at $z \sim 1$ are marginally detectable. Detectability increases rapidly with decreasing distance because of the decrease of optical depth as well as increase of the original (unattenuated) flux, and $\mathrm{TeV}$ gamma-rays from $z \sim 0.5$ would be easily detectable.

The above estimate is based on the relatively small distance scale of GRBs, $z_{\max } \sim 1$, but larger distance scales are also suggested by cosmic evolution of star formation rate (Totani 1997, 1998; Sahu et al. 1997; Wijers et al. 1998) or by the recently detected host galaxy for GRB971214 (Kulkarni et al. 1998). The original TeV flux expected on the Earth before absorbed in intergalactic fields is almost insensitive to the unknown distance scale of GRBs, because we have just scaled the total energy $(E)$ from the observed energy emitted as GRB photons. The increase of the intergalactic optical depth with $z$ beyond $z \sim 1$ is also rather slow compared to $z<1$ (Salamon \& Stecker 1998), and hence the detectability of $\mathrm{TeV}$ gamma-rays is not so sensitive to the GRB distance scale, if $z_{\max } \gtrsim 1$. More precise estimate of detectability requires better determination of infrared background, and in other words, discovery of the $\mathrm{TeV}$ afterglow would give important infomation for the intergalactic infrared photon field.

\section{Detectability of GeV Photons}

GRB940217 has the third-largest energy fluence in the 4B BATSE catalog (Paciesas et al. 1997), and this GRB is famous for the detection of high energy photons by the EGRET detector with very long duration (Hurley et al. 1994). The EGRET detected high energy photons ranging from $36 \mathrm{MeV}$ to $18 \mathrm{GeV}$ during $\sim 5000$ seconds. We show that this EGRET photons are well explained by the proton synchrotron of the model. The observer's 
time when the external shock phase begins, $t_{d}$, is given by $r_{d} /\left(2 \gamma_{0}^{2}\right)$, where the deceleration radius is $r_{d}=\left(17 b E / 16 \pi n m_{p} \gamma_{0}^{2}\right)^{1 / 3}$. This deceleration time sensitively depends on $\gamma_{0}$, and it can be as short as $t_{d}=1.3 b_{200}^{1 / 3} E_{52}^{1 / 3} n_{1}^{-1 / 3} \gamma_{1000}^{-8 / 3}$ sec when $\gamma_{0} \sim 1000$. Therefore the EGRET photons can be considered as the external shock origin and our model is applicable, although other explanations by internal shocks may also be possible. The observed photon spectrum (Fig. 3 of Hurley et al. 1994) seems consistent with the standard spectrum of synchrotron radiation, $d n / d \varepsilon_{\gamma} \propto \varepsilon_{\gamma}^{-3 / 2}$, and by fitting the data with this photon index we find that the differential photon flux $d n / d \varepsilon_{\gamma}$ is $\sim 2 \times 10^{-11}$ and $2 \times 10^{-12} \varepsilon_{\gamma, \mathrm{GeV}}^{-3 / 2}$ photons $\mathrm{cm}^{-2} \mathrm{sec}^{-1} \mathrm{keV}^{-1}$ for the first $180 \mathrm{sec}$ and delayed photons (180-5400 sec), respectively. This time evolution is consistent with the $t^{-3 / 4}$ profile of Eq. (3). If we assume that the fluence of this GRB in the BATSE range, $6.6 \times 10^{-4} \mathrm{erg} \mathrm{cm}^{-2}$, is $1 / 2000$ of the total energy $E$, the distance to this GRB is $d=113 b_{200}^{1 / 2} E_{52}^{1 / 2} \mathrm{Mpc}$ and hence the differential photon flux obtained from Eq. (3) is $5.6 \times 10^{-11} \xi_{p} \xi_{B}^{3 / 4} E_{52}^{1 / 4} b_{200}^{1 / 4} n_{1}^{1 / 2} t_{5}^{-3 / 4} \varepsilon_{\gamma, \mathrm{GeV}}^{-3 / 2}$ photons $\mathrm{cm}^{-2} \mathrm{sec}^{-1} \mathrm{keV}^{-1}$, where $t_{5}=t_{\mathrm{obs}} /(5000$ $\mathrm{sec})$. This photon flux is consistent with the observation if the energy conversion into accelerated protons and magnetic field is near the equipartition: $\xi_{p} \sim \xi_{B}^{3 / 4} \sim 0.2 n_{1}^{-1 / 4}$. Therefore the delayed GeV photons from GRB940217 are naturally explained by our model. On the other hand, there exist some GRBs which are as bright as the GRB940217 but not accompanied by such long-duration GeV photons. In such GRBs, the onset of external shock phase might be very long after the GRBs and/or the density of interstellar matter is quite low. In fact, if the progenitor of a GRB is a massive star, the intensive stellar wind prior to the death of the star could have swept up the interstellar medium near the star. In this case the $\mathrm{TeV}$ or $\mathrm{GeV}$ luminosity which is proportional to $n^{1 / 2}$ could be very small and significantly delayed compared to GRBs.

Because of the $e^{ \pm}$pair-creation in intergalactic field, more than $99 \%$ of $\mathrm{TeV}$ gamma-rays from $z \sim 1$ must disappear before reaching the Earth. The created $e^{ \pm}$pairs, whose energy is about $\sim \mathrm{TeV}$, lose their energy by the inverse-Compton (IC) scattering of the cosmic microwave background photons and typical energy of the secondary IC photons is $\varepsilon_{2} \sim 0.6 \varepsilon_{\gamma, \mathrm{TeV}}^{2} \mathrm{GeV}$, which is in the detectable range of the EGRET. The expected time delay of these secondary photons is $\sim d /\left(2 c \gamma_{\text {pair }}^{2}\right)=1.8 d_{3} \gamma_{6}^{-2}$ day (Cheng \& Cheng 1996), where $\gamma_{\text {pair }}=10^{6} \gamma_{6}$ is the Lorentz factor of created pair. If the attenuation of $\mathrm{TeV}$ gamma-rays is significant $\left(e^{\tau} \gg 1\right)$, almost all energy originally emitted in $\mathrm{TeV}$ range should be converted into $\mathrm{GeV}$ range, which is much larger than the original energy emitted in $\mathrm{GeV}$ photons by proton synchrotron. This effect becomes significant with increasing optical depth for $\mathrm{TeV}$ photons and compensate the decrease of flux due to the increase of distance, and hence we might be able to detect delayed $\mathrm{GeV}$ photons for rather distant GRBs. In the limit of $e^{\tau} \gg 1$ and neglecting the time delay due to propagation in the intergalactic field, we have estimated the differential photon flux as 
$1.7 \times 10^{-7} \xi_{p} \xi_{B}^{3 / 4} E_{52}^{5 / 4} b_{200}^{5 / 4} n_{1}^{1 / 2} t_{\text {day }}^{-3 / 4} d_{3}^{-2} \varepsilon_{2, \mathrm{GeV}}^{-7 / 4}$ photons $\mathrm{GeV}^{-1} \mathrm{~cm}^{-2} \mathrm{~s}^{-1}$. This estimate may be further reduced by the delay of $\sim$ a few days, but not so far from the EGRET sensitivity. Delayed $\mathrm{GeV}$ emission on a time scale of a few days from GRBs at cosmological distances may be detectable by the EGRET, or is likely to be detected by the future GLAST experiment. Future ground-based telescopes with reduced threshold energy down to tenth of $\mathrm{GeV}$ range, e.g., the VERITAS project (Weekes et al. 1998) will also be useful for search of the secondary $\mathrm{GeV}$ photons.

\section{Discussion}

The typical Lorentz factor of electrons in afterglow shock is $\gamma_{e}=\xi_{e}\left(m_{p} / m_{e}\right) \gamma$ and we have considered the case of $\xi_{e} \sim 1 / 2000$. Then the observed synchrotron photon energy of a electron with a Lorentz factor $\gamma_{e}$ is $\gamma \gamma_{e}^{2} e B / m_{e}=2.5 \times 10^{-4}\left(2000 \xi_{e}\right)^{2} \xi_{B}^{1 / 2} b_{300}^{1 / 2} E_{52}^{1 / 2} t_{\text {day }}^{-3 / 2} \mathrm{eV}$, which is the radio band, and it seems to contradict the observations of X-ray or optical afterglows. However acceleration of electrons and/or IC scattering of synchrotron photons can raise the photon energy. Furthermore, $\xi_{e}$ may also increase with time in afterglow. In fact, $\tau_{e p} /(r / \gamma)=870 b_{200}^{-1 / 3} E_{52}^{-1 / 3} n_{1}^{-2 / 3} \gamma^{8 / 3}$ decreases as $\propto \gamma^{8 / 3} \propto t^{-1}$, and it is possible that energy transfer from protons into electrons becomes efficient gradually with the expansion of afterglow shock. Note that there are considerable variations for the behavior of afterglows observed in X, optical, and radio bands (e.g., Groot et al. 1998). Efficiency of energy transfer into electrons and its time evolution could have large variations among GRBs and it may be one of the origins of the complicated behavior of GRB afterglows.

It should be noted that the proton synchrotron emission extends to X-ray, optical, and radio bands with the standard synchrotron spectrum of $d L / d \varepsilon_{\gamma} \propto \varepsilon^{(1-\alpha) / 2}$. If $\alpha=2$, luminosity per decade of photon energy at $\varepsilon_{\gamma}=1 \mathrm{keV}$ is $\sqrt{10^{9}}$ times smaller than that in the $\mathrm{TeV}$ range, and the differential flux observed from a distance of 3000 $\mathrm{Mpc}$ is $\sim 1.5 \times 10^{-14} \xi_{p} \xi_{B}^{3 / 4} E_{52}^{5 / 4} b_{200}^{5 / 4} n_{1}^{1 / 2} t_{\text {day }}^{-3 / 4} \varepsilon_{\gamma, \mathrm{keV}}^{-1 / 2} d_{3}^{-2} \mathrm{erg} \mathrm{cm}^{-2} \mathrm{sec}^{-1} \mathrm{keV}^{-1}$. This flux is comparable to the observed flux of X-ray afterglows for distant bursts such as GRB970402 or GRB970508 (Piro et al. 1997a,b). Therefore the proton synchrotron radiation could contribute to the X-ray afterglows, although it is rather difficult to detect in the optical or lower energy bands due to the hardness of the spectrum. Note that optical afterglows are associated only to a small fraction of GRBs for which X-ray afterglows are detected. Therefore it can be speculated that proton synchrotron was dominant in such GRBs. The complicated behavior of afterglows may be a consequence of complicated mixture of proton synchrotron and electron synchrotron or inverse Compton scattering.

We finally note that energy emitted as $10^{20-21} \mathrm{eV}$ protons must be roughly the same 
with that emitted as GRB photons, if the GRB is the origin of ultra high energy cosmic rays (UHECRs) observed on the Earth (Waxman 1995; Vietri 1995). On the other hand, in our model, energy distributed to such protons is much greater (at least by a factor of $\sim 100$ ) than GRB photons. However, as we have shown, such protons are likely trapped in afterglow shock and lose their energy by synchrotron radiation. If the escape fraction of protons just cancels the overproduction of $10^{20} \mathrm{eV}$ protons, GRBs could still be the origin

of UHECRs. If the escape fraction is further smaller, then the UHECRs must be explained by other sources. The Larmor radius becomes larger with increasing proton energy, and the escape fraction may increase with proton energy. This suggests a possibility that the spectrum of UHECRs becomes significantly harder above $10^{20} \mathrm{eV}$, which should be tested by future experiments.

The author would like to thank S. Sasaki and an anonymous referee for useful comments. He has been supported by the Research Fellowships of the Japan Society for the Promotion of Science for Young Scientists, and the Grant-in-Aid for the Scientific Research Fund (No. 3730) of the Ministry of Education, Science, and Culture of Japan.

\section{REFERENCES}

Blandford, R. D. and McKee, C. F. 1976, Phys. Fluids 19, 1130

Blinnikov, S. I. et al. 1984, Sov. Astr. Lett. 10, 177

Böttcher, M. \& Dermer, C. D. 1998, preprint, astro-ph/9801027 (to appear in ApJ Lett.)

Cheng, L. X. \& Cheng, K. S. 1996, ApJ, 459, L79

Connaughton, V. et al. 1997, ApJ, 479, 859

Groot, P. J. et al. 1998, ApJ, 493, L27

Hurley, K. et al. 1994, Nature, 372, 652

Katz, J. I. 1994, ApJ, 432, L107

Kifune, T. 1996, Space Sci. Rev., 75, 31

Kulkarni, S. R. et al. 1998, Nature, 393, 35

Lifshitz, E.M. and Pitaevskii, L.P. 1981, Physical Kinetics (Oxford: Pergamon Press).

Lipunov, V. M. et al. 1995, ApJ, 454, 593 
Mészáros, P. \& Rees, M. 1997, ApJ, 476, 232

Metzger M. R. et al. 1997, Nature, 387, 879

Paciesas, W. S. et al. 1997, 4B BATSE catalog is available at http://cossc.gsfc.nasa.gov/cossc/BATSE.html

Paczyński, B. 1998, ApJ, 494, L45

Paczyński, B. \& Rhoads, J. 1993, ApJ, 418, L5

Piran, T. 1994, in Unsolved Problems in Astrophysics, ed. J.N. Bahcall and J.P. Ostriker (New Jersey: Princeton University Press), 343

Piro, L. et al. 1997a, IAU Circ. 6617

Piro, L. et al. 1997b, IAU Circ. 6656

Salamon, M.H. \& Stecker, F. W. 1998, ApJ, 493, 547

Sahu, K. et al. 1997, ApJ, 489, L127

Totani, T. 1997, ApJ, 486, L71

Totani, T. 1998, submitted to ApJ, and available on astro-ph.

Vietri, M. 1995, ApJ, 453, 883

Vietri, M. 1997a, ApJ, 478, L9

Vietri, M. 1997b, ApJ, 488, L105

Vietri, M. 1997c, Phys. Rev. Lett. 78, 4328

Waxman, E. 1995, Phys. Rev. Lett. 75, 386

Waxman, E. 1997a, ApJ, 487, L5

Waxman, E. 1997b, ApJ, 489, L33

Weekes T. C. et al. 1998, to be published in proceedings of ACT Workshop, Kruger Park.

Wijers, A. M. J. R., Rees, M. J. \& M'eszáros, P. 1997, MNRAS, 288, L51

Wijers, R. M. J., Bloom, J. S., Bagla, J. S. \& Natarajan, P. 1998, MNRAS, 294, L17 